\title{
The Parsec-Scale Central Components of FR I Radio Galaxies
}

\author{
Preeti Kharb \& Prajval Shastri \\ Indian Institute of Astrophysics, Koramangala, Bangalore - 34, India.
}

\begin{abstract}
We present results of our study of the parsec-scale components of radio galaxies as observed at radio, optical and X-ray wavelengths, in the framework of the Unified Scheme.
\end{abstract}

BACKGROUND : A majority of a complete sample of 3CR FR I radio galaxies and $\sim 1 / 2$ of the 3CR FR II radio galaxies, imaged with the HST show Compact Central Cores (CCCs). We show that the optical flux density of the CCCs, $F_{o}$, in FR Is is correlated with the radio core prominence, $R_{c}$ (at $5 \mathrm{GHz}$ ). If the core is the unresolved relativistically beamed jet and the lobes are unbeamed then $R_{c}=S($ core $) / S($ extended $)$ becomes a statistical measure of orientation. We further examine the correlation of the CCC emission with the jet-to-counterjet surface brightness ratio $R_{j}=B(j e t) / B($ cjet $)$, where differences in the surface brightness between the jet and counterjet at a given distance from the nucleus are interpreted as effects of Doppler beaming and dimming respectively, on intrinsically symmetrical flows. The CCC $F_{o}$ in FR I radio galaxies is well correlated with $R_{j}$ (at a distance of a few pc from core). These correlations support the argument of Chiaberge, Capetti, \& Celotti (1999), that the CCC radiation is of a non-thermal synchrotron origin, which is relativistically beamed along with the radio emission. We used the CCC data of 27 3CR FR Is in Chiaberge et al. 1999; NGC 7052 and NGC 6251 in Capetti \& Celotti (1999) and Hardcastle \& Worrall (2000), respectively; 26 3CR FR IIs in Chiaberge, Capetti \& Celotti (2000) and BL Lacs in Capetti \& Celotti (1999). The $R_{j}$ values come from the VLBI observations of Giovannini et al. 1999 and references therein. The X-ray core flux densities, $F_{x}$ are from the ROSAT data in Hardcastle \& Worrall (2000).

RESULTS : Optical CCCs in FR I radio galaxies : The CCC optical emission is well correlated with $R_{c}$, the significance of the correlation being at the 0.001 level (Spearman rank test: See Figure 1a). This correlation suggests that the CCC flux density is orientation dependent in the same way as the core radio emission and it may thus constitute the optical counterpart of the radio synchrotron jet. In Figure 1a we have included a set of BL Lacs, whose extended radio luminosities span the same range as the FR Is, for comparison. The BL Lacs extend the correlation to higher $R_{c}$, as would be expected if FR Is are the parent population of BL Lacs. The $F_{o}$ of CCCs and $R_{j}$ for the FR I radio galaxies, show a correlation significant at the 0.01 level (Spearman rank test; Figure 1b), furtier substantiating the claim that the optical CCC is a part of the jet.

Optical CCCs in FR II radio galaxies : The optical core flux density for the sample of FR II radio galaxies also shows a correlation with $R_{c}$, although weaker (significance level 0.1; Spearman rank test). See Figure 2a. 
$X$-ray core components in radio galaxies : We examine the correlation between the X-ray core flux density, $F_{x}$ (nJy) and radio core prominence, $R_{c}$ (Figure $2 \mathrm{~b}$ ). The correlation is significant at the 0.2 level for FR Is taken alone while it is significant at the 0.1 level for FR I and FR IIs taken together.
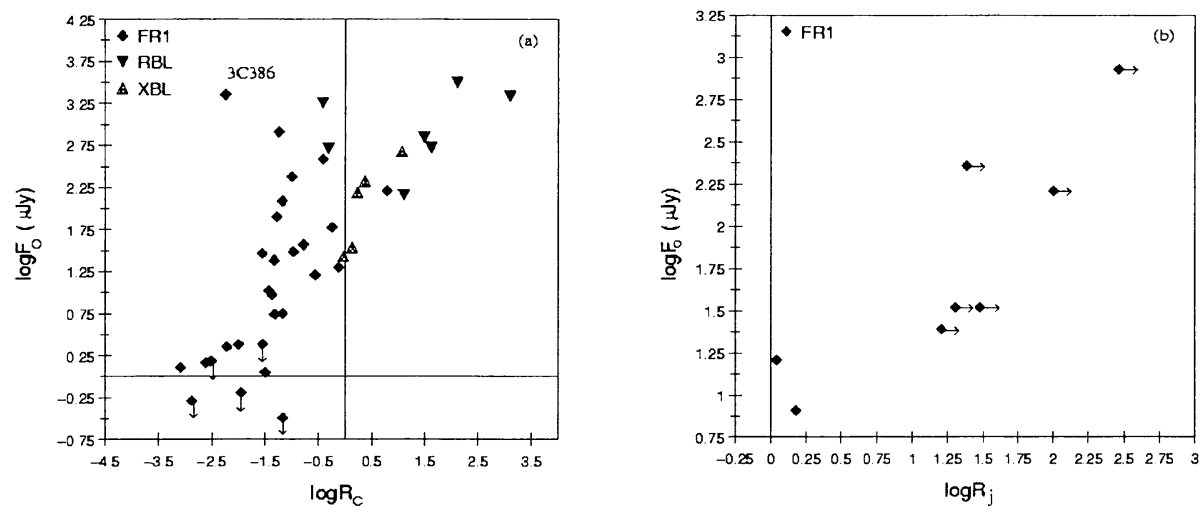

Figure 1. (a). $\quad R_{c}$ vs $F_{o}(\mu \mathrm{Jy})$ for $\mathrm{FR}$ I radio galaxies and $\left(\mathrm{RBL}=\right.$ radio-selected $\& \mathrm{XBL}=\mathrm{X}$-ray selected) $\mathrm{BL}$ Lacs (b). $R_{j}$ vs $F_{o}$ $(\mu \mathrm{Jy})$ for FR I radio galaxies. (Arrows denote upper limits).
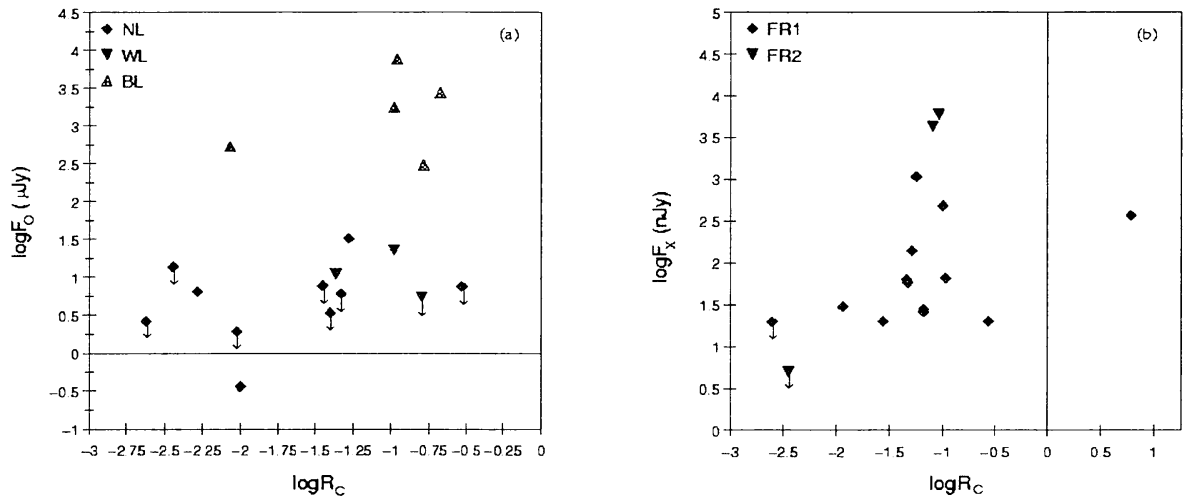

Figure 2. (a). $R_{c}$ vs $F_{o}(\mu \mathrm{Jy})$ for FR II (NL=Narrow-line, $\mathrm{BL}=$ Broad-line, $\mathrm{WL}=$ Weak-line) radio galaxies (b). $R_{c}$ vs $F_{x}$ (nJy) for FR I and FR II radio galaxies. (Arrows denote upper limits).

\section{References}

Capetti, A., \& Celotti, A. 1999, MNRAS, 304, 434

Chiaberge, M., Capetti, A., \& Celotti, A. 1999, A\&A, 349, 77

Chiaberge, M., Capetti, A., \& Celotti, A. 2000, A\&A, 355, 873

Giovannini, G., Taylor, G. B., Arbizzani, E., Bondi, M., Cotton, W. D., Feretti, L., Lara, L., \& Venturi, T. 1999, ApJ, 522,101

Hardcastle, M. J., \& Worrall, D. M. 2000, MNRAS, 314, 359 